\title{
High Diagnostic Yield of Targeted Next-Generation Sequencing in a Cohort of Patients With Congenital Hypothyroidism Due to Dyshormonogenesis
}

OPEN ACCESS

Edited by:

Noriyuki Koibuchi,

Gunma University, Japan

Reviewed by:

Izuki Amano,

Gunma University, Japan

Hiroaki Shimizu,

Dokkyo Medical University, Japan

*Correspondence: Michel Polak michel.polak@aphp.fr Aurore Carré

aurore.carre@inserm.fr

${ }^{\dagger}$ These authors share last authorship

Specialty section: This article was submitted to Thyroid Endocrinology, a section of the journal

Frontiers in Endocrinology

Received: 15 April 2020 Accepted: 15 December 2020 Published: 22 February 2021

Citation:

Stoupa A, Al Hage Chehade G,

Chaabane $R$, Kariyawasam $D$, Szinnai G, Hanein S, Bole-Feysot C, Fourrage $C$, Nitschke $P$

Thalassinos C, Pinto G, Mnif $M$, Baron S, De Kerdanet M, Reynaud $R$ Barat P. Hachicha M, Belquith N,

Polak M and Carré A (2021) High Diagnostic Yield of Targeted Next-Generation Sequencing in a Cohort of Patients With

Congenital Hypothyroidism Due to Dyshormonogenesis.

Front. Endocrinol. 11:545339. doi: 10.3389/fendo.2020.545339

\begin{abstract}
Athanasia Stoupa ${ }^{1,2,3}$, Ghada Al Hage Chehade ${ }^{3}$, Rim Chaabane ${ }^{4}$, Dulanjalee Kariyawasam ${ }^{3}$, Gabor Szinnai ${ }^{5,6}$, Sylvain Hanein ${ }^{7}$, Christine Bole-Feysot ${ }^{8}$, Cécile Fourrage ${ }^{9}$, Patrick Nitschke ${ }^{9}$, Caroline Thalassinos ${ }^{3}$, Graziella Pinto ${ }^{3}$, Mouna Mnif ${ }^{10}$, Sabine Baron ${ }^{11}$, Marc De Kerdanet ${ }^{12}$, Rachel Reynaud ${ }^{13}$, Pascal Barat ${ }^{14}$, Mongia Hachicha ${ }^{15}$, Neila Belguith ${ }^{4,16}$, Michel Polak ${ }^{1,2,3,17,18^{* t}}$ and Aurore Carré ${ }^{1,2 * t}$

1 INSERM U1016, Cochin Institute, Faculté de Médecine, Université Paris Descartes, Sorbonne Paris Cité, Paris, France, 2 IMAGINE Institute affiliate, Paris, France, ${ }^{3}$ Pediatric Endocrinology, Gynecology and Diabetology Unit, Hôpital Universitaire Necker-Enfants Malades, AP-HP, Paris, France, ${ }^{4}$ Laboratory of Human Molecular Genetics, Medicine School, University of Sfax, Sfax, Tunisia, ${ }^{5}$ Pediatric Immunology, Department of Biomedicine, University of Basel, Basel, Switzerland, 6 Pediatric Endocrinology, University Children's Hospital Basel, University of Basel, Basel, Switzerland, ${ }^{7}$ INSERM U1163, IMAGINE Institute, Translational Genetics, Université Paris Descartes, Sorbonne Paris Cité, Paris, France, ${ }^{8}$ Genomics Platform, INSERM UMR 1163, Paris Descartes Sorbonne Paris Cite University, Imagine Institute, Paris, France, ${ }^{9}$ Bioinformatics Platform, Paris Descartes University, IMAGINE Institute, Paris, France, ${ }^{10}$ Endocrinology Department, CHU Hedi Chaker, Sfax, Tunisia, ${ }^{11}$ Pediatrics Department, CHU Nantes, Nantes, France, ${ }^{12}$ Pediatrics Department, CHU Rennes, Rennes, France, ${ }_{13}$ Pediatrics Department, CHU La Timone, Marseille, France, ${ }^{14} \mathrm{CHU}$ de Bordeaux, Pediatric Endocrinology, Bordeaux, France, ${ }^{15}$ Pediatrics Department, CHU Hedi Chaker, Sfax, Tunisia, ${ }^{16}$ Medical Genetics Department, CHU Hedi Chaker, Sfax, Tunisia, ${ }^{17}$ Centre de Référence des Maladies Endocriniennes Rares de la Croissance et du Développement, Necker-Enfants Malades University Hospital, Paris, France, ${ }^{18}$ Centre Régional de Dépistage Néonatal (CRDN) lle de France, Paris, France
\end{abstract}

Objective: To elucidate the molecular cause in a well-characterized cohort of patients with Congenital Hypothyroidism $(\mathrm{CH})$ and Dyshormonogenesis $(\mathrm{DH})$ by using targeted next-generation sequencing (TNGS).

Study design: We studied 19 well-characterized patients diagnosed with $\mathrm{CH}$ and $\mathrm{DH}$ by targeted NGS including genes involved in thyroid hormone production. The pathogenicity of novel mutations was assessed based on in silico prediction tool results, functional studies when possible, variant location in important protein domains, and a review of the recent literature.

Results: TNGS with variant prioritization and detailed assessment identified likely disease-causing mutations in 10 patients (53\%). Monogenic defects most often involved TG, followed by DUOXA2, DUOX2, and NIS and were usually homozygous or compound heterozygous. Our review shows the importance of the detailed phenotypic description of patients and accurate analysis of variants to provide a molecular diagnosis.

Conclusions: In a clinically well-characterized cohort, TNGS had a diagnostic yield of $53 \%$, in accordance with previous studies using a similar strategy. TG mutations were the 
most common genetic defect. TNGS identified gene mutations causing $\mathrm{DH}$, thereby providing a rapid and cost-effective genetic diagnosis in patients with $\mathrm{CH}$ due to $\mathrm{DH}$.

Keywords: congenital hypothyroidism, dyshormonogenesis, mutations, targeted next-generation sequencing, gland in situ

\section{INTRODUCTION}

Congenital hypothyroidism $(\mathrm{CH})$ is the most common neonatal endocrine disorder, with an incidence of $1 / 2,500-3,500$ newborns $(1,2)$. Among patients with $\mathrm{CH}, 65 \%$ have thyroid dysgenesis (TD), with a large phenotypic spectrum encompassing athyreosis, thyroid ectopy, hypoplasia of an orthotopic gland, and hemithyroid (3). In the remaining 35\% of patients, $\mathrm{CH}$ is due to dyshormonogenesis $(\mathrm{DH})$ with a thyroid gland in situ (GIS) with or without goiter. DH may lead to goiter formation due to thyroid tissue overstimulation by increased TSH levels. Most cases of DH are due to mutations in TG, TPO, SLC5A5/NIS, SLC26A4/PDS, IYD/DEHAL1, DUOX2, DUOXA2, DUOX1, DUOXA1, and SLC26A7, which are involved in thyroid hormone production and usually inherited on an autosomal recessive basis (4-6).

The proportion of patients with $\mathrm{CH}$ due to $\mathrm{DH}$ who receive a molecular diagnosis varies widely across studies, from 20 to $60 \%$ (7-13). Factors contributing to this variability include differences in patient phenotypes, clinical characterization of the patients (imaging techniques, as thyroid ultrasound and scintigraphy, perchlorate test, thyroglobulin measurement), geographic origin, and mainly variant classification.

The objective of this study was to assess the diagnostic yield of targeted Next Generation Sequencing (TNGS) in a cohort of 19 well-characterized patients with $\mathrm{CH}$ due to $\mathrm{DH}$. We also report the results of an extensive literature review of studies describing genetic findings in patients with $\mathrm{CH}$, with special attention to those having DH.

\section{MATERIALS AND METHODS}

\section{Patients}

Nineteen patients with permanent primary $\mathrm{CH}$, referred by various centers in France, Tunisia $(n=2)$, and the United States of America (USA) $(n=1)$ were included in the study. Diagnosis of primary $\mathrm{CH}$ was based on systematic newborn screening in France and increased venous TSH at control blood sample. Cut-off in blood spot for newborn screening is $15 \mathrm{mIU} / \mathrm{L}$ in France and $20 \mathrm{mIU} / \mathrm{L}$ in New York (USA); no newborn screening is available in Tunisia. $\mathrm{CH}$ was diagnosed at birth for all patients except the patient from USA and the Tunisian patients diagnosed during the first months or year of life, respectively, due to clinical symptoms suggesting hypothyroidism and confirmed by high TSH levels and low

Abbreviations: CH, congenital hypothyroidism; DH, dyshormonogenesis; GIS, gland in situ; MAF, minor allele frequency; NGS, Next generation Sequencing. free T4 levels (FT4), according to laboratory reference values. Inclusion criteria of 19 patients were $\mathrm{CH}$ with GIS and at least one of the following: clinical goiter $(\geq 2 S D)$, available thyroid scintigraphy providing an evaluation of thyroid position and radionuclide uptake, and a perchlorate discharge test performed. This study was approved by French Biomedecine Agency. Written, informed consent was obtained from the individuals and minors' legal guardian for the publication of any potentially identifiable images or data included in this article.

\section{Detection of Mutations}

We designed a TNGS panel (HypothySeq NGS) of 78 genes including genes known to be associated with $\mathrm{CH}$ (thyroid dysgenesis; dyshormonogenesis; thyroid hormone transport protein defects; and inborn errors in thyroid hormone membrane transport, metabolism, or action) and candidate genes that have been identified in animal models (mouse and zebrafish knock-out models) or by microarray assays but not yet validated in humans. Sensitivity (false-negative rate) of the panel was assessed in positive controls with known thyroid disease-causing mutations, including mutations in dyshormonogenesis genes, and specificity (false-positive rate) in healthy individuals previously screened using whole exome sequencing for another research project.

TNGS on HiSeq 2500 system (Illumina, San Diego, CA) and bioinformatics analyses were already described (14). Mean coverage for each gene is reported Table $\mathbf{1}$.

\section{Prioritizing Strategy for Filtering Pathogenic Variants}

Variants (including frameshift mutations, missense and nonsense mutations, and splicing-site mutations) identified in known dyshormonogenesis genes were considered for the analysis. If available, functional data and segregation analysis results were taken into consideration.

Inheritance is recessive for TPO, TG, DUOX2, DUOXA2, SLC5A5, and SLC26A4 and dominant for PAX8. Genome variations were defined using PolyDiag in-house software for

TABLE 1 | Reference transcript and average coverage of genes responsible for $\mathrm{CH}$ and $\mathrm{DH}$.

\begin{tabular}{llr}
\hline & Reference transcript & $\begin{array}{r}\text { Average } \\
\text { coverage }\end{array}$ \\
\hline TG & NM_003235 & 614 \\
TPO & NM_000547 & 694 \\
DUOX2 & NM_014080 & 586 \\
DUOXA2 & NM_207581 & 516 \\
NIS/SLC5A5 & NM_000453 & 577 \\
PDS/SLC26A4 & NM_000441 & 720 \\
DEHAL1/IYD & NM_001164694, & 580 \\
& NM_203395 &
\end{tabular}


TNGS, which filters out irrelevant and common polymorphisms based on frequencies extracted from the following public databases: US National Center for Biotechnology Information database of single nucleotide polymorphisms (SNP) (dbSNP), 1000 Genomes, Exome Variant Server and Exome Aggregation Consortium (ExAC). Consequences of mutations on protein function were predicted using three algorithms: Polyphen2, SIFT, and Mutation Taster. Mutations were ranked based on the impact of each variant predicted by combined annotationdependent depletion (CADD) then compared using the mutation significance cut-off, which is a gene-level specific cutoff for CADD scores.

For deletions or insertions in exons, information on the variants was sought in similar published studies. Sanger sequencing was performed to validate and segregate the identified variants (3500xL Genetic Analyzer, Thermo Fisher Scientific, Waltham, MA). Primer sequences are shown in Table 2.

\section{RESULTS}

\section{Clinical Description of the Study Cohort}

We studied 19 index cases of $\mathrm{CH}$ due to dyshormonogenesis (Table 3). Among them, 16 were born in France [two of them (patients \#4 and \#18) born in France to consanguineous parents, and of Moroccan and Turkish origin, respectively] and were diagnosed with primary $\mathrm{CH}$ at birth by routine neonatal screening (TSH cutoff, $15 \mathrm{mIU} / \mathrm{L}$ ) followed by a venous-blood TSH assay. CH was diagnosed at day 75 of age in one patient (\#19) born in the USA and at 2 months and 5 months of age in two patients (\#14 and \#15 respectively) born in Tunisia. These three patients underwent evaluation for clinical symptoms suggesting hypothyroidism and were found to have high TSH levels and low FT4 levels. Of the 19 patients, eight had a family history of $\mathrm{CH}$ and five were born to consanguineous parents, including one with an affected sibling (\#1). A goiter was evident at diagnosis in nine patients including two (\#3 and \#16) diagnosed with goiter in utero. Fetal goiter was diagnosed during one of the routine fetal sonograms performed in France during pregnancy at 13,22 , and 32 gestational weeks. In our cases, the diagnosis was made on the second ultrasound, around 22 gestational week and treated with intraamniotic levothyroxine injections, mean dose between 200 and 400 micrograms/ injection. The response to levothyroxine treatment was assessed by decrease in thyroid size in fetal ultrasound and/or normalization of fetal thyroid hormones. According to the European Society for Paediatric Endocrinology guidelines (15) the FT4 level at diagnosis indicated severe $\mathrm{CH}$ in nine patients $(\mathrm{FT} 4<5 \mathrm{pmol} / \mathrm{L})$ and moderate $\mathrm{CH}$ in 1 patient (FT4, 10-15 $\mathrm{pmol} / \mathrm{L})$. Thyroglobulin was not assayed routinely. A perchlorate discharge test was performed in 14 patients, of whom 11 had a positive result $>10 \%$.

\section{Genetic Results and Diagnostic Yield}

TGNS allowed the molecular diagnosis in the majority of patients (10/19) screened, providing a diagnostic yield of 53\% (Table 4, Figures 1-3). We identified of 14 novel variants on 24. Twelve/24 variants were causative in function of the context (genetic model, specificity of variants). TG was the most common site of mutations, followed by DUOXA2 for causative variants (Figure 1). Figure 2 shows the familial pedigrees and Figure 3 the location of DH-causing mutations.

\section{TG Variants}

Seven novel variants have been identified by TNGS in our study cohort, located mainly in the type 1 repeat protein domain and the acetylcholinesterase (ACHE)-like domain.

Two siblings (patient \#1 and his brother) were compound heterozygous for two previously described TG mutations: an insertion in a splice site (c.638+1 G>A) leading to exon 5 skipping and a missense mutation (c.886C $>$ T, pR277X) producing a premature stop codon (16, 17). Familial segregation and Sanger sequencing confirmed that one variant was transmitted by the mother and the other one by the father (Table 4). Another known homozygous $T G$ mutation (c.6701C >A, p.A2215D) in the ACHE-like domain, associated with deficient TG secretion due to TG retention within the endoplasmic reticulum $(18,19)$ was identified in patient \#2. Unfortunately, no parental DNA was available. Patient \#3 was compound heterozygous for two $T G$ mutations including the previously described c.4588 C>T mutation (inherited from the father) that produces a premature stop codon (p.R1511X) with skipping of the exon 22 domain (34). Exon 22 skipping may result in protein structure alterations responsible for protein retention within the cell. This nonsense mutation occurs in a CpG dinucleotide sequence and may be caused by deamination of a methylated cytosine resulting in a thymine (21). The other

TABLE 2 | Primers sequences to verify variants by PCR and Sanger sequencing.

\begin{tabular}{|c|c|c|c|}
\hline Gene & Exon & Primer Forward & Primer Reverse \\
\hline PAX8 & 3 & GGCTCTGGCTAAATCCCTGTCTAA & TCCCTGCCTGATTGTTCAGCAT \\
\hline PAX8 & 7 & TGCAGGAAGGTCGGCTTGTT & GACAGCCAGCCAAGCTCTTCA \\
\hline SLC5A5 & 9_10 & GATGGTGTGGACGGTCTCTC & TAATGGGAAAGAGGGAAAGG \\
\hline TG & 5 & GAGTGCATATGCTGCTCGAC & TCAAGGTGAGTGTGGGCTG \\
\hline $\mathrm{TG}$ & 6 & TTCCTITCACTAGGCGTGG & GCAGGCAGTCACTCTAGCTG \\
\hline TG & 7 & AACTITGAAACCCAAGAGGC & AGGTCAGGGCTTCCПтCTG \\
\hline TG & 9 & СTCTGTGCCAGAAGATGTGG & CTGTACTGCATTGGGTCAGG \\
\hline TG & 22 & TAGGAGTCAGGGGATTCCAG & AGCCCTTGAGACTACTCCCC \\
\hline TG & 26 & TCСAACTCTGCCATGTITG & CAGCTCCATGTTGTGTGTCC \\
\hline
\end{tabular}


TABLE 3 | Clinical description of cohort of patients with $\mathrm{CH}$ due to $\mathrm{DH}$.

\begin{tabular}{|c|c|c|c|c|c|c|c|c|c|c|c|c|c|}
\hline Patient & $\begin{array}{l}\text { Country } \\
\text { of origin }\end{array}$ & $\begin{array}{l}\text { Consanguineous } \\
\text { parents }\end{array}$ & Age / Sex & $\begin{array}{c}\text { Age at } \\
\text { diagnosis }\end{array}$ & $\begin{array}{c}\text { TSH at } \\
\text { diagnosis } \\
\text { (mlU/L) }\end{array}$ & $\begin{array}{l}\text { free } \\
\text { T4 at }\end{array}$ & Thyroglobulin & $\begin{array}{l}\text { Goiter at } \\
\text { diagnosis }\end{array}$ & $\begin{array}{l}\text { Cervical } \\
\text { Ultrasound }\end{array}$ & $\begin{array}{c}\text { Thyroid } \\
\text { Scintigraphy }\end{array}$ & $\begin{array}{l}\text { Perchlorate } \\
\text { test }\end{array}$ & $\begin{array}{l}\text { Associated } \\
\text { features }\end{array}$ & Familial cases \\
\hline 1 & France & No & 19 y / M & D9 & 470 & 3.5 & NA & No & NA & $\begin{array}{l}\text { Normal position - } \\
\text { elevated uptake }\end{array}$ & $11 \%$ & - & $\begin{array}{l}\mathrm{CH} \text { in a brother } \\
\text { with similar } \\
\text { clinical features }\end{array}$ \\
\hline 2 & France & No & 30 y / M & D19 & 49 & NA & 1.5 & Yes & $\begin{array}{l}\text { Normal position } \\
\text { - RL 46×25 mm } \\
\text { - LL 45×30 mm }\end{array}$ & $\begin{array}{l}\text { Normal position - } \\
\text { elevated uptake }\end{array}$ & $\mathrm{N}$ & - & - \\
\hline 3 & France & No & $6 y / M$ & D19 & 143 & 8.4 & NA & $\begin{array}{l}\text { Yes. In } \\
\text { utero }\end{array}$ & NA & $\begin{array}{c}\text { Normal position, } \\
\text { normal uptake }\end{array}$ & $10.4 \%$ & - & $\begin{array}{l}\text { Subclinical non- } \\
\text { autommune } \\
\text { hypothyroidism } \\
\text { in mother and } \\
\text { grand-mother }\end{array}$ \\
\hline 4 & Morocco & Yes & $9 y / F$ & D19 & 100 & NA & 2,512 & Ye, mild & $\begin{array}{l}\text { Normal position } \\
\text { - RL 18×9 mm - } \\
\text { LL 20x10 mm }\end{array}$ & $\begin{array}{l}\text { Normal position - } \\
\text { elevated uptake }\end{array}$ & $84 \%$ & - & - \\
\hline 5 & France & No & $19 y / M$ & D14 & 657 & 3 & NA & No & NA & $\begin{array}{l}\text { Normal position - } \\
\text { elevated uptake }\end{array}$ & $50 \%$ & - & - \\
\hline 6 & Tunisia & Yes & $11 \mathrm{y} / \mathrm{M}$ & D4 & 637 & & undetectable & NA & Yes & Normal position & $\begin{array}{c}\text { Normal } \\
\text { position, } \\
\text { normal } \\
\text { uptake }\end{array}$ & N & - \\
\hline
\end{tabular}

\begin{tabular}{|c|c|c|c|c|c|c|c|c|c|c|c|c|c|}
\hline 7 & France & No & $6 y / F$ & D8 & 443 & 6.1 & 1.2 & No & $\begin{array}{l}\text { Normal position } \\
\text { - RL 21×10 mm } \\
- \text { - LL } 17 \times 10 \mathrm{~mm}\end{array}$ & Normal position & $13 \%$ & - & - \\
\hline 8 & France & No & $24 \mathrm{y} / \mathrm{F}$ & D3 & 110 & 6.2 & NA & No & Normal position & $\begin{array}{l}\text { Normal position - } \\
\text { elevated uptake }\end{array}$ & $42 \%$ & - & - \\
\hline 9 & France & No & $22 \mathrm{y} / \mathrm{M}$ & D3 & high (NA) & NA & NA & Yes, mild & Normal position & NA & NA & - & $\begin{array}{c}2 \text { brothers with } \\
\mathrm{CH} \text { and similar } \\
\text { clinical } \\
\text { presentation }\end{array}$ \\
\hline 10 & France & No & $17 \mathrm{y} / \mathrm{F}$ & D13 & 580 & $<1.8$ & NA & Yes & $\begin{array}{l}\text { Normal position } \\
\text { RL 30×6 mm - } \\
\text { LL 30×3.5 mm }\end{array}$ & No lodine uptake & - & - & - \\
\hline 11 & France & No & $3 y / M$ & D10 & 300 & 14 & NA & No & NA & $\begin{array}{c}\text { Normal position - } \\
\text { normal uptake }\end{array}$ & $50 \%$ & $\begin{array}{l}\text { Unilateral } \\
\text { cryptorchidism } \\
\text { - no renal } \\
\text { anomalies }\end{array}$ & $\begin{array}{c}\mathrm{CH} \text { and gland } \\
\text { in situ (mother) } \\
\text { - urinary tract } \\
\text { duplication } \\
\text { (father) }\end{array}$ \\
\hline
\end{tabular}


TABLE 3 | Continued

\begin{tabular}{|c|c|c|c|c|c|c|c|c|c|c|c|c|c|}
\hline Patient & $\begin{array}{l}\text { Country } \\
\text { of origin }\end{array}$ & $\begin{array}{l}\text { Consanguineous } \\
\text { parents }\end{array}$ & Age / Sex & $\begin{array}{c}\text { Age at } \\
\text { diagnosis }\end{array}$ & $\begin{array}{c}\text { TSH at } \\
\text { diagnosis } \\
\text { (mlU/L) }\end{array}$ & $\begin{array}{l}\text { free } \\
\text { T4 at }\end{array}$ & Thyroglobulin & $\begin{array}{r}\text { Goiter at } \\
\text { diagnosis }\end{array}$ & $\begin{array}{l}\text { Cervical } \\
\text { Ultrasound }\end{array}$ & $\begin{array}{c}\text { Thyroid } \\
\text { Scintigraphy }\end{array}$ & $\begin{array}{l}\text { Perchlorate } \\
\text { test }\end{array}$ & $\begin{array}{l}\text { Associated } \\
\text { features }\end{array}$ & Familial cases \\
\hline 12 & France & No & $7 \mathrm{y} / \mathrm{F}$ & D9 & 53.8 & NA & 431 & No & $\begin{array}{l}\text { Normal position } \\
\text { - length: RL } 21 \\
\text { mm - LL } 21 \mathrm{~mm}\end{array}$ & $\begin{array}{c}\text { Normal position - } \\
\text { normal uptake }\end{array}$ & $25 \%$ & & - \\
\hline 13 & France & No & $8 y / F$ & D9 & 111.5 & $<5$ & NA & No & $\begin{array}{c}\text { Normal position } \\
\text { - RL } 7.7 \times 5 \mathrm{~mm} \\
\text { - LL } 8.1 \times 5.9 \\
\mathrm{~mm}\end{array}$ & Normal position & $57 \%$ & - & $\begin{array}{c}\mathrm{CH} \text { in the twin } \\
\text { sister }\end{array}$ \\
\hline 14 & Tunisia & Yes & $32 \mathrm{y} / \mathrm{M}$ & $2 \mathrm{M}$ & 90 & 2 & 35 & No & Normal position & No lodine uptake & NA & - & $\begin{array}{l}\mathrm{CH} \text { (brother. } \\
\text { similar clinical } \\
\text { presentation) - } \\
\mathrm{CH} \text { (uncle) }\end{array}$ \\
\hline 15 & Tunisia & Yes & $24 \mathrm{y} / \mathrm{F}$ & $5 \mathrm{M}$ & $>60$ & $<0.5$ & NA & No & Normal position & No lodine uptake & NA & - & - \\
\hline 16 & France & No & $15 \mathrm{y} / \mathrm{M}$ & D1 & 180 & 4.9 & NA & $\begin{array}{l}\text { Ye, in } \\
\text { utero }\end{array}$ & Normal position & Normal position & $26 \%$ & - & - \\
\hline 17 & France & No & 26 y / M & D3 & 37.6 & 20 & 71 & Yes & $\begin{array}{c}\text { Normal position } \\
-\mathrm{RL} 6 \times 4 \times 11 \\
\mathrm{~mm}-\mathrm{LL} 4 \times 5 \times 10 \\
\mathrm{~mm}\end{array}$ & $\begin{array}{l}\text { Normal position - } \\
\text { normal uptake }\end{array}$ & $\mathrm{N}$ & - & $\mathrm{CH}$ in the sister \\
\hline 18 & Turkey & Yes & 9 y/F & D3 & 591 & 3.5 & 2,110 & Yes & 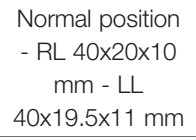 & Normal position & $27 \%$ & - & \\
\hline 19 & $\begin{array}{l}\text { United } \\
\text { States }\end{array}$ & No & $5 \mathrm{y} / \mathrm{F}$ & D75 & 755 & 1.2 & NA & No & Normal position & $\begin{array}{l}\text { Normal position - } \\
\text { reduced uptake }\end{array}$ & NA & $\begin{array}{l}\text { Prematurity. } \\
\text { No intrauterine } \\
\text { growth }\end{array}$ & $\begin{array}{c}\mathrm{CH} \text { in the twin } \\
\text { sister }\end{array}$ \\
\hline \multirow[t]{5}{*}{$\begin{array}{l}\text { Normal } \\
\text { values }\end{array}$} & Age & TSH (mlU/L) & T4 (pmol/L) & & & & & & & & & & \\
\hline & $2-7 D$ & $1.1-15.6$ & $11.6-36.0$ & & & & & & & & & & \\
\hline & 8- $15 \mathrm{D}$ & $0.87-7.8$ & $9.5-28.9$ & & & & & & & & & & \\
\hline & $16-30 \mathrm{D}$ & $0.82-6.9$ & $9.3-23.5$ & & & & & & & & & & \\
\hline & $\begin{array}{c}1 \text { month } \\
-1 y\end{array}$ & $0.80-6.05$ & $8.3-18.6$ & & & & & & & & & & \\
\hline $\begin{array}{l}\text { Normal } \\
\text { values }\end{array}$ & TG & 20-70ng/mL & & & & & & & & & & & \\
\hline
\end{tabular}

NA, not available; N, normal; $D$, days; $M$, months; $y$, years; $M$, male; F, female; RL, right lobe; LL, left lobe. Normal perchlorate discharge test is considered when the discharge is less than $10 \%$. 
TABLE 4 | Molecular and protein descriptions regarding variants found in patients with $\mathrm{CH}$ due to $\mathrm{DH}$.

\begin{tabular}{|c|c|c|c|c|c|c|c|c|c|c|c|c|c|c|c|}
\hline Patient & Gene & cDNA change & Amino acid & Exon & Homozygous (ho)/ & Variant & Protein & Variant name - ExAC & & In silico $\mathrm{p}$ & prediction & & Inheritance & Reference/ho & Causative \\
\hline & & & & & & & & & SIFT & $\begin{array}{l}\text { Polyphen- } \\
2\end{array}$ & $\begin{array}{l}\text { Mutation } \\
\text { Taster }\end{array}$ & $\begin{array}{l}\text { CADD } \\
\text { score }\end{array}$ & & & \\
\hline 1 & TG & c. $638+1 \mathrm{G}>\mathrm{A}$ & & 1_ex.5 & $\mathrm{He}$ & $\begin{array}{l}\text { splice } \\
\text { donor } \\
\text { region }\end{array}$ & Type 1 repeat & 8_133885467_G_A- 0 & - & - & - & 25.9 & $\begin{array}{l}\text { carrier } \\
\text { mother }\end{array}$ & $\begin{array}{l}\text { Alzahrani et al. } \\
\text { (16) / ho / no }\end{array}$ & Yes \\
\hline & TG & c.886 C>T & p.R277X & 7 & $\mathrm{He}$ & $\begin{array}{c}\text { premature } \\
\text { stop } \\
\text { codon }\end{array}$ & Type 1 repeat & $\begin{array}{l}\text { rs121912648 - } 0.0003625- \\
\text { gnomAD } 0.0003535\end{array}$ & - & - & - & 37 & $\begin{array}{l}\text { carrier } \\
\text { father }\end{array}$ & $\begin{array}{c}\text { Van de graaf } \\
\text { et al. (17)/ ho / } \\
\text { no }\end{array}$ & Yes \\
\hline 2 & TG & c. $6701 \mathrm{C}>\mathrm{A}$ & p.A2215D & 38 & Ho & missense & $\begin{array}{l}\text { ACHE-like } \\
\text { domain }\end{array}$ & $\begin{array}{l}\text { rs370991693 - } 0.00004141- \\
\text { gnomAD } 0.00004245\end{array}$ & Deleterious & Damaging & $\begin{array}{l}\text { Disease } \\
\text { causing }\end{array}$ & 32 & NA & $\begin{array}{l}\text { Caputo et al. } \\
\text { (18), Pardo } \\
\text { et al. } 2009 \\
\text { (19)/ het } \\
\text { compound and } \\
\text { ho/ deficient } \\
\text { TG secretion, } \\
\text { retention in } \\
\text { cells }\end{array}$ & Yes \\
\hline & DUOX2 & c.601_602insG & p.G202Tfs99 & 6 & $\mathrm{He}$ & frameshift & $\begin{array}{c}\mathrm{N} \text {-terminal } \\
\text { peroxidase-like } \\
\text { domain }\end{array}$ & $\begin{array}{c}\text { rs565500345 - } 0.001 \text { - gnomAD } \\
0.00009553\end{array}$ & - & - & - & -1 & NA & $\begin{array}{c}\text { Pfarr et al. (20)- } \\
\text { het compound/ } \\
\text { No }\end{array}$ & No \\
\hline 3 & TG & c.2132_2133insG & p.A693Gfs24 & 9 & $\mathrm{He}$ & frameshift & Type 1 repeat & 8_133899750_A_AG-0 & - & - & - & -1 & & No & Yes \\
\hline & TG & c. $4588 \mathrm{C}>\mathrm{T}$ & p.R1511X & 22 & $\mathrm{He}$ & $\begin{array}{l}\text { premature } \\
\text { stop }\end{array}$ & Type 1 repeat & $\begin{array}{l}\text { rs121912646 - } 0.00006591 \\
\text {-gnomAD 0.00009195 }\end{array}$ & - & - & - & 38 & $\begin{array}{l}\text { carrier } \\
\text { father }\end{array}$ & $\begin{array}{l}\text { Targovnik et al. } \\
(21) \text {, ho/ No }\end{array}$ & Yes \\
\hline & SLC26A4 & C. $199 A>C$ & p.T67P & 3 & $\mathrm{He}$ & missense & - & 7_107303775_A_C - 0 & Deleterious & Benign & Polymorphism & 8.8 & & No & No \\
\hline & DUOX2 & c. $598 \mathrm{G}>\mathrm{A}$ & p.G200R & 6 & $\mathrm{He}$ & missense & $\begin{array}{l}\mathrm{N} \text {-terminal } \\
\text { peroxidaselike } \\
\text { domain }\end{array}$ & $\begin{array}{c}\text { rs2467827 - } 0.003 \text {-gnomAD } \\
0.0009541\end{array}$ & Tolerated & $\begin{array}{l}\text { Possibly } \\
\text { damaging }\end{array}$ & $\begin{array}{l}\text { Disease } \\
\text { causing }\end{array}$ & 11.2 & & No & No \\
\hline 4 & DUOX2 & c. $3155 \mathrm{G}>\mathrm{A}$ & p.C1052Y & 24 & & missense & - & $\begin{array}{l}\text { rs76343591 - } 0.004218 \\
\text {-gnomAD } 0.001294\end{array}$ & Deleterious & $\begin{array}{l}\text { Possibly } \\
\text { damaging }\end{array}$ & Polymorphism & 24.7 & NA & $\begin{array}{l}\text { Tonacchera } \\
\text { et al. (22)/ het } \\
\text { compound / } \\
\text { partial defect in } \\
\mathrm{H} 2 \mathrm{O} 2 \\
\text { production }\end{array}$ & Yes \\
\hline & TG & $\begin{array}{l}\text { c.5299_5301 } \\
\text { del.GAT }\end{array}$ & p.D1748del & 27 & $\mathrm{He}$ & $\begin{array}{c}\text { no } \\
\text { frameshift }\end{array}$ & Type 3 repeat & $\begin{array}{c}\text { rs112749206 - } 0.011 \text {-gnomAD } \\
0.004399\end{array}$ & - & - & & - & NA & $\begin{array}{c}\text { Brust et al. } \\
(23) / \text { het } \\
\text { compound / } \\
\text { No }\end{array}$ & No \\
\hline & TG & c. $5370 A>G$ & p. $11771 \mathrm{M}$ & 27 & $\mathrm{He}$ & missense & Type 3 repeat & $\begin{array}{c}\text { rs } 73710715-0.011 \text {-gnomAD } \\
0.004414\end{array}$ & Benign & Tolerated & Polymorphism & 0 & NA & No & No \\
\hline 5 & TG & c.648_649insG & p.A198Gfs14 & 6 & $\mathrm{He}$ & frameshift & Type 1 repeat & 8_133894118_T_TG - 0 & - & - & - & -1 & $\begin{array}{l}\text { carrier } \\
\text { mother }\end{array}$ & No & Yes \\
\hline & TG & c.4588 C>T & p.R1511X & 22 & $\mathrm{He}$ & $\begin{array}{l}\text { premature } \\
\text { stop } \\
\text { codon }\end{array}$ & Type 1 repeat & $\begin{array}{l}\text { rs121912646 - } 0.00006591 \\
\text {-gnomAD 0.00009195 }\end{array}$ & - & - & - & 38 & $\begin{array}{l}\text { carrier } \\
\text { father }\end{array}$ & $\begin{array}{l}\text { Targovnik et al. } \\
(21) / \text { ho /No }\end{array}$ & Yes \\
\hline 6 & TG & c. $7859 \mathrm{G}>\mathrm{A}$ & p.G2601D & 45 & $\mathrm{He}$ & missense & $\begin{array}{l}\text { ACHE-like } \\
\text { domain }\end{array}$ & rs978923522 - 0 & Tolerated & Benign & Polymorphism & 9.9 & NA & No & No \\
\hline
\end{tabular}


TABLE 4 | Continued

\begin{tabular}{|c|c|c|c|c|c|c|c|c|c|c|c|c|c|c|c|}
\hline Patient & Gene & cDNA change & $\begin{array}{l}\text { Amino acid } \\
\text { change }\end{array}$ & Exon & $\begin{array}{l}\text { Homozygous (ho)/ } \\
\text { Heterozygous (he) }\end{array}$ & $\begin{array}{l}\text { Variant } \\
\text { type }\end{array}$ & $\begin{array}{l}\text { Protein } \\
\text { Domain }\end{array}$ & $\begin{array}{l}\text { Variant name - EXAC } \\
\text { frequence/dbSNP or MAF and } \\
\text { allele frequency in gnomAD }\end{array}$ & & In silico $\mathrm{p}$ & diction & & Inheritance & $\begin{array}{l}\text { Reference/ho } \\
\text { or het/Func- } \\
\text { tional study }\end{array}$ & Causative \\
\hline \multirow[t]{2}{*}{7} & TG & $c .886 \mathrm{C}>\mathrm{T}$ & p.R277X & 7 & $\mathrm{He}$ & $\begin{array}{l}\text { premature } \\
\text { stop } \\
\text { codon }\end{array}$ & Type 1 repeat & $\begin{array}{l}\text { rs121912648 - } 0.0003625 \\
\text {-gnomAD } 0.0003535\end{array}$ & - & - & & 37 & $\begin{array}{l}\text { carrier } \\
\text { father }\end{array}$ & $\begin{array}{c}\text { Van de graaf } \\
\text { et al. (17)/ ho / } \\
\text { No }\end{array}$ & Yes \\
\hline & TG & c. $5182 \mathrm{~T}>\mathrm{C}$ & p.C1709R & 26 & $\mathrm{He}$ & missense & Type 3 repeat & rs37600169 - 0.00002471 & Deleterious & Damaging & $\begin{array}{l}\text { Disease } \\
\text { causing }\end{array}$ & 25.2 & $\begin{array}{l}\text { carrier } \\
\text { mother }\end{array}$ & No & Yes \\
\hline \multirow[t]{4}{*}{8} & DUOXA2 & c. $205+2 T>C$ & & 2_ex2 & $\mathrm{He}$ & $\begin{array}{l}\text { essential } \\
\text { splicing }\end{array}$ & $\begin{array}{c}\| \\
\text { transmembrane } \\
\text { domain }\end{array}$ & $\begin{array}{l}\text { rs201506037 - } 0.00038 \\
\text {-gnomAD } 0.0004583\end{array}$ & - & - & & 25.2 & NA & No & Yes \\
\hline & DUOXA2 & c. $463 C>G$ & p.L155V & 4 & $\mathrm{He}$ & coding & $\begin{array}{l}\text { extracellular } \\
\text { domain }\end{array}$ & $\begin{array}{l}\text { rs201808443 }-0.00040 \\
\text {-gnomAD } 0.0004595\end{array}$ & Deleterious & Damaging & $\begin{array}{l}\text { Disease } \\
\text { causing }\end{array}$ & 24.7 & NA & No & Yes \\
\hline & TG & c.2233_2234insT & p.L727Afs ${ }^{\star} 3$ & 10 & $\mathrm{He}$ & frameshift & Type 1 repeat & $\begin{array}{c}\text { eva_exac_8_133900286_C_CT } \\
-0\end{array}$ & - & - & - & -1 & NA & No & $?$ \\
\hline & TG & c.3452delT & p.V1132Afs ${ }^{\star 31}$ & 16 & $\mathrm{He}$ & frameshift & Type 1 repeat & rs766130576- 0.00003 & - & - & - & & NA & No & ? \\
\hline 9 & TPO & c. $866 \mathrm{~T}>\mathrm{C}$ & p.F289S & 8 & $\mathrm{He}$ & missense & $\begin{array}{l}\text { Heme } \\
\text { peroxidase }\end{array}$ & 2_1480904_T_C - 0 & Deleterious & Damaging & $\begin{array}{l}\text { Disease } \\
\text { causing }\end{array}$ & 25.2 & & No & No \\
\hline 10 & TPO & c.1768+1insGT & CTGCCAG & 1_ex10 & $\mathrm{He}$ & $\begin{array}{l}\text { splice } \\
\text { donor } \\
\text { region }\end{array}$ & $\begin{array}{l}\text { Heme } \\
\text { peroxidase }\end{array}$ & $\begin{array}{c}\text { 2_1491764_G_GGTCTGC CAG } \\
-0\end{array}$ & - & - & & -1 & & No & No \\
\hline 11 & PAX8 & c. $101 \mathrm{~T}>\mathrm{A}$ & p. $134 \mathrm{~N}$ & 3 & $\mathrm{He}$ & missense & $\begin{array}{l}\text { Paired box } \\
\text { protein }\end{array}$ & 2_114004421_A_T_0.00008331 & Deleterious & Damaging & $\begin{array}{l}\text { Disease } \\
\text { causing }\end{array}$ & 28.2 & $\begin{array}{l}\text { carrier } \\
\text { mother }\end{array}$ & $\begin{array}{l}\text { Lanzerath et al. } \\
\text { (24)/ thyroid } \\
\text { hypoplasia / } \\
\text { het compound } \\
\text { / No }\end{array}$ & Yes \\
\hline 12 & PAX8 & $c .397 C>T$ & p.R133W & 5 & $\mathrm{He}$ & missense & $\begin{array}{l}\text { Paired box } \\
\text { protein }\end{array}$ & 2_114000348_G_A - 0 & Deleterious & Damaging & $\begin{array}{l}\text { Disease } \\
\text { causing }\end{array}$ & 35 & NA & $\begin{array}{c}\text { Vincenzi et al. } \\
\text { (25) / het / Yes } \\
\text { but not } \\
\text { deleterious on } \\
\text { TG }\end{array}$ & No \\
\hline 13 & PAX8 & c. $658 \mathrm{C}>\mathrm{T}$ & p.R220X & 7 & $\mathrm{He}$ & $\begin{array}{l}\text { premature } \\
\text { stop } \\
\text { codon }\end{array}$ & - & 2_113999247_G_A - 0 & - & - & & 37 & $\begin{array}{l}\text { carrier } \\
\text { mother }\end{array}$ & $\begin{array}{l}\text { Fu et al. (26) / } \\
\text { het hypoplasia } \\
\text { / No }\end{array}$ & No \\
\hline 14 & SLC5A5 & C. $1183 \mathrm{G}>\mathrm{A}$ & p.G395R & 10 & Ho & missense & $\begin{array}{c}\mathrm{X} \\
\text { transmembrane } \\
\text { domain }\end{array}$ & $\begin{array}{l}\text { rs121909180 - } 0.00006595 \\
\text {-gnomAD } 0.00004597\end{array}$ & Deleterious & Damaging & $\begin{array}{l}\text { Disease } \\
\text { causing }\end{array}$ & 33 & $\begin{array}{l}\text { carrier } \\
\text { mother, } \\
\text { father NA }\end{array}$ & $\begin{array}{l}\text { Kosugi et al. } \\
\text { (27)/ ho / no } \\
\text { iodide uptake }\end{array}$ & Yes \\
\hline 15 & SLC5A5 & c. $1183 \mathrm{G}>\mathrm{A}$ & p.G395R & 10 & Ho & missense & $\begin{array}{c}x \\
\text { transmembrane } \\
\text { domain }\end{array}$ & $\begin{array}{l}\text { rs 121909180 - } 0.00006595 \\
\text {-gnomAD 0.00004597 }\end{array}$ & Deleterious & Damaging & $\begin{array}{l}\text { Disease } \\
\text { causing }\end{array}$ & 33 & $\begin{array}{l}\text { carrier } \\
\text { father and } \\
\text { mother }\end{array}$ & $\begin{array}{l}\text { Kosugi et al. } \\
(27) / \text { ho / no } \\
\text { iodide uptake }\end{array}$ & Yes \\
\hline
\end{tabular}

NA, Not available.

? No definitive molecular diagnosis.

For TG, the amino acid positions are numbered after subtracting the 19-amino acid signal peptide. 

GIS, normal size
$\mathrm{n}=10$

\section{TNGS including TG, TPO, DUOX2, DUOXA2, SLC5A5/NIS} SLC26A4/PDS, DEHAL1/IYD, PAX8

19 patients with $\mathrm{CH}$ and $\mathrm{DH}$

Filtering variants: $\mathrm{MAF}<0.02$, coding region, splice site<smiles>C1CCCCC1</smiles>

24 identified variants:

$12 / 24$ causative variants $(50 \%)$

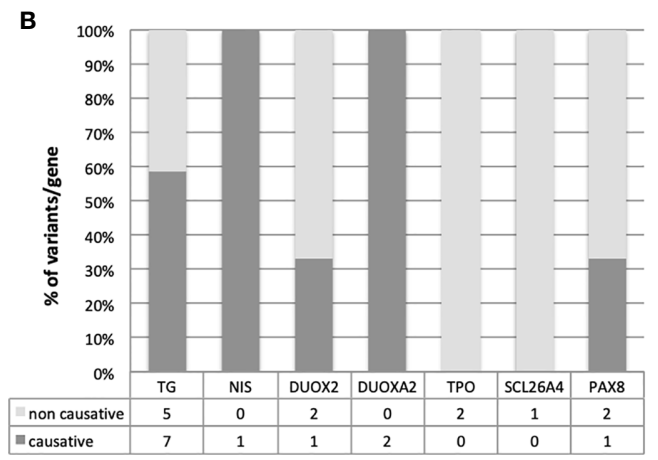

C

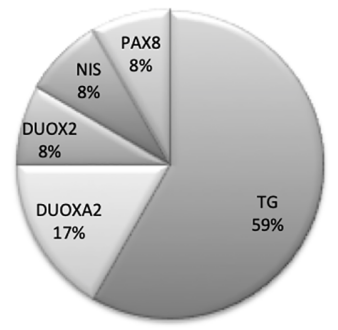

$\%$ of causative variants of each gene among total causative variants

FIGURE 1 | Schematic representation of found variants in the cohort of patients with $\mathrm{CH}$ due to $\mathrm{DH}$. (A) Flowchart of the selection and distribution of variants identified by TNGS in 19 patients, including TG, TPO, DUOX2, DUOXA2, SLC5A5/NIS, SLC26A4, DEHAL1/IYD, and PAX8 (B) Distribution of variants detected for each gene and absolute numbers of non-causative and causative variants. (C) Distribution of causative variants for each gene calculated relative to the total number of causative variants.
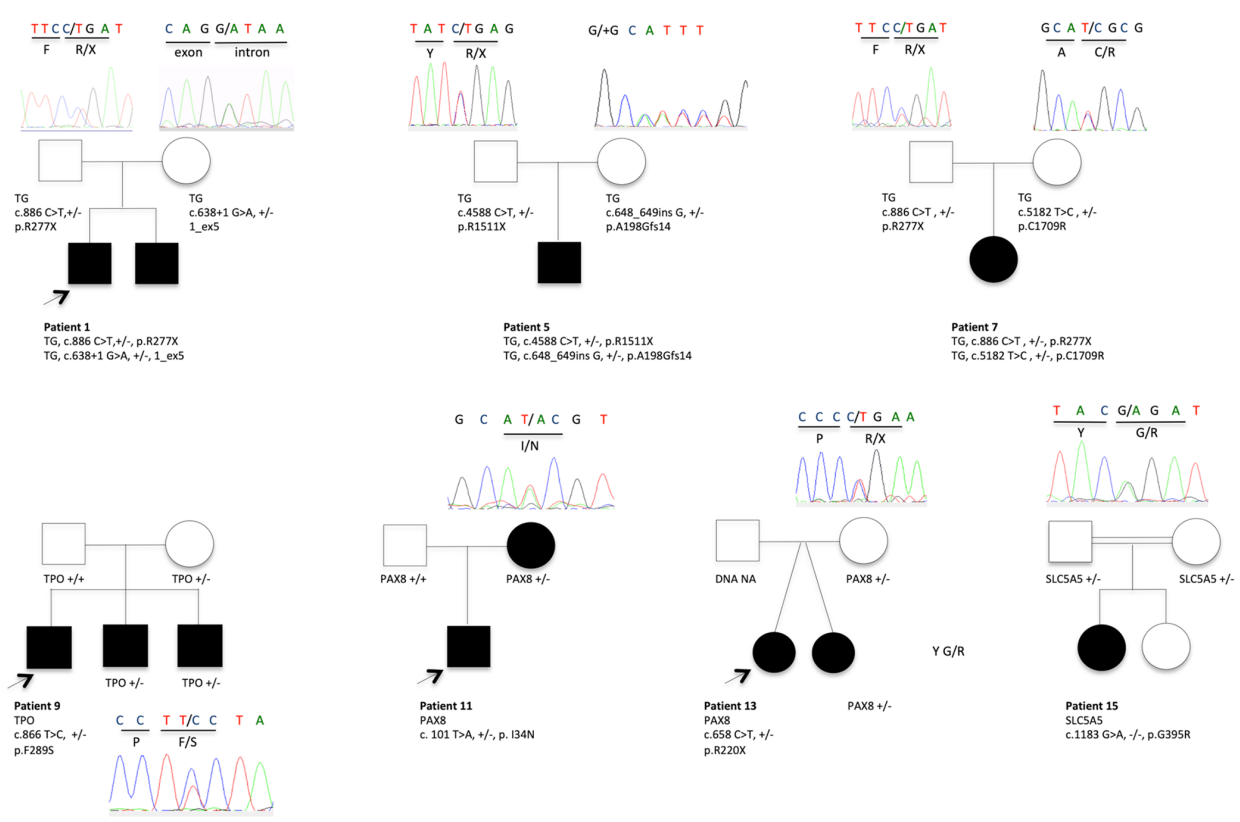

FIGURE 2 | Pedigrees showing causative variant distribution and segregation in seven families. The patients with $\mathrm{CH}$ and $\mathrm{DH}$ are represented by filled boxes. In familial forms, the index patient is indicated by an arrow. Representative chromatograms are shown for each family member. NA, not available for DNA sampling. 
TG

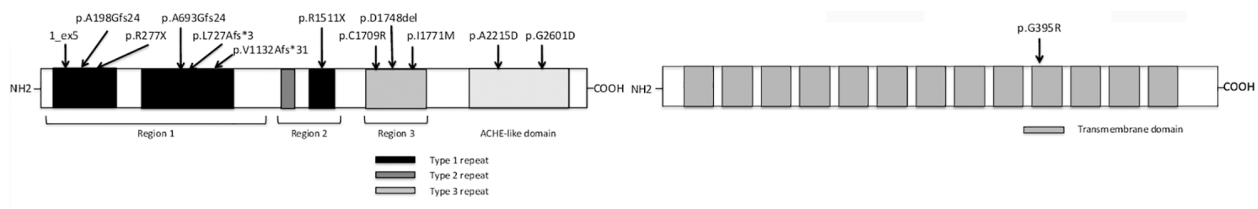

DUOX2

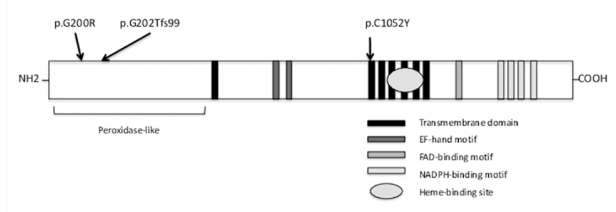

TPO

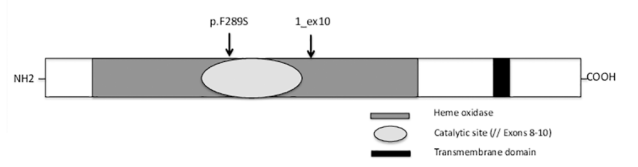

DUOXA2

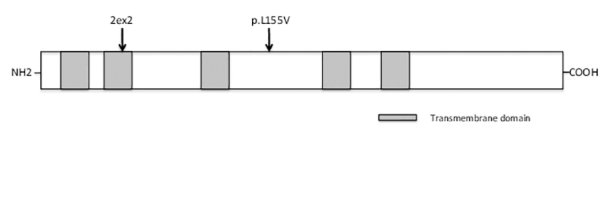

PAX8

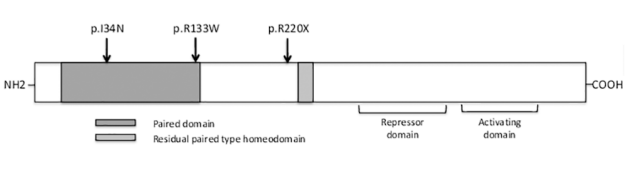

FIGURE 3 | Variants identified in the present study with their location in the protein domains of thyroglobulin (TG), sodium-iodide symporter (NIS), dual oxidase 2 (DUOX2), dual oxidase maturation factor 2 (DUOXA2), thyroid peroxidase (TPO), and paired-box protein 8 (PAX8). TG: arrows show the positions of identified mutations in key structural domains including the repetitive type 1, type 2, and type 3 cysteine-rich regions (shown in black, dark gray, and light gray boxes, respectively) and follows the acetylcholinesterase homology (ACHE-like) domain (adapted from Di Jeso and Arvan) (28). NIS: one identified variant (arrows) in 1 of 13 different transmembrane domains (adapted from Kosugi et al.) (29). DUOX2: the different protein domains are as follows: the peroxidase-like domain is in the Nterminal region and the black boxes represent the transmembrane domains, the dark-gray boxes the EF-hand motifs, the light gray boxes the FAD-binding domain, the narrow white boxes the NADPH-binding domains, and the oval box the heme-binding site (adapted from Moreno et al.) (30). Mutations are shown by arrows in the various protein regions. DUOXA2: light gray boxes represent transmembrane domains (adapted from Grasberger and Refetoff) (31) and mutations are shown by arrows. TPO: the heme oxidase domain is shown in light gray with an oval representing its catalytic site (encoded by exons 8-10) and the transmembrane domain is represented in dark gray at the C-terminal end of the protein (adapted from Deladoey et al.) (32). The two TPO mutations are shown by arrows in the heme oxidase domain. PAX8: paired domain (dark gray box), residual paired type homeodomain (light gray box), and the repressor and activating domains defined by dark lines (adapted from Poleev et al.) (33). The three PAX8 variants are shown by arrows.

TG mutation was a novel variant, c.2132_2133 insG, in exon 9, responsible for a frameshift in the Type-1 domain. This c.2132_2133 insG mutation explains therefore along with the p.R1511X, the clinical phenotype of the patient. Patient \#5 also had two TG mutations. One (c.4588 C>T, p.R1511X) was a previously described mutation (34) for which no functional studies are available, inherited from the father, and responsible for a stop codon. The other, inherited from the mother, was a novel $G$ insertion in exon 6 leading to a frameshift in the Type 1 TG domain and to a premature stop codon. The previously reported c.886 C>T mutation (p.R277X), also found in patient $\# 1$, was identified in patient \#7 along with a novel missense mutation that replaces a cysteine with an arginine (c.5182 $\mathrm{T}>\mathrm{C}$, p.C1709R), located in the type 3 repeat domain of TG, and responsible for absence of a disulfide bond that may alter the conformation of TG. A novel TG monoallelic variant (c.7859G>A, p.G2601D) located in the ACHE-like domain was found in another patient (patient \#6); its pathogenicity cannot be ascertained in silico.

\section{DUOX2 and DUOXA2 Variants}

TGNS identified one case (patient \#4) with a probably causative biallelic DUOX2 mutation leading to partial deficiency in $\mathrm{H}_{2} \mathrm{O}_{2}$ production (22). Interestingly this patient had also two heterozygous $T G$ variants located in the same type-3 protein domain, of which one-a 3-bp (GAT) deletion at amino acid position 1,767-has been reported previously (23) and the other is novel (c.5370A $>$ G, p.I1771M); suggesting that accumulation of pathogenic variants may lead to $\mathrm{CH}$.

In our study cohort, patients \#2 and \#3 besides TG mutations, carried a monoallelic DUOX2 variant not located in DUOX2 functional domains or hot-spot mutations. The DUOX2 variant of patient \#2 has been previously described in patients with $\mathrm{DH}$, without any functional study (20).

The accumulation of variants in different $\mathrm{DH}$ genes applies on other cases, as in patient \#8 heterozygous for four novel variants in TG and DUOXA2. The two TG variants, c.2233_2234insT and c.3452delT, were in the type 1 repeat domain. One DUOXA2 variant, c.205+2 T>C, was in an essential splice site. In silico predictions predictive algorithms show an abolishment of natural splice donor site, suggesting a splicing effect of the variation. The other DUOXA2 mutation, c.463 C>G, p.L155V, was a missense variant in the larger extracellular domain. The patient had a GIS and a positive perchlorate discharge test. Although the two DUOXA2 mutations may explain the organification defect, a pathogenic effect of the two TG mutations cannot be ruled out, particularly as no TG assay was performed at diagnosis. 


\section{TPO Variants}

We did not identify any biallelic mutations but only two novel monoallelic variants. Monoallelic TPO mutations have been reported to cause DH with showed monoallelic expression (35). The first TPO variant c.866 T>C, p.F289S, is in exon 8, encoding for the catalytic domain (patient \#9). In silico prediction tools suggested a deleterious or disease-causing effect. Monoallelic expression of the TPO mutation in patient $\# 9$ cannot be ruled out, as no thyroid tissue study was performed. Familial segregation for patient \# 9 showed that mother carried this TPO variant despite a normal thyroid function. The second variant (patient \#10) is an insertion (c.1768+1insGTCTGCCAG), after exon 10. This variation does not affect the main splice donor site, but because of the duplicated sequence it creates a putative new splice donor site, which is the same as the previous site. Its pathogenicity remains questionable, as ideally functional data would be helpful.

\section{PAX8 Variants}

PAX8 mutations have been chiefly described in patients with $\mathrm{CH}$ and thyroid dysgenesis, some of whom also have renal and urinary malformations (36). However, mutations in the PAX8 binding domain have been found in patients with $\mathrm{CH}$ and $\mathrm{DH}$ characterized by defective iodide organification and positive perchlorate discharge test or partial iodide transport defect, with normal-sized thyroid gland $(37,38)$. Three previously reported variants have been identified in three cases of cohort; two of them in the DNA binding domain. One case (patient \#11), with GIS and a positive perchlorate discharge test, was heterozygous for a mutation located in the binding domain of PAX8 (c.101T>A, p.I34N). This mutation has been reported previously in a patient with $\mathrm{CH}$ and thyroid gland hypoplasia (24), who was also heterozygous for another PAX8 mutation (p.V35I), in the DNA binding domain. Although no functional data are available, given its location in the binding domain, the p.I34N mutation probably impairs transactivation of TG or TPO. Moreover, this variant was inherited from the mother, who also had $\mathrm{CH}$ and GIS. Patient \#12 was heterozygous for a missense variant (c.397C>T, p.R133W) at the end of the PAX8 DNA binding domain. This previously described mutation had no deleterious effect on TG transactivation or with the synergism between PAX8 and NKX2-1 (25). Pathogenicity is unclear, for the third variant (patient \#13) (c.658C>T, p.R220X) located after the PAX8 binding domain and responsible for a premature stop codon; described previously in a single patient who did not undergo functional studies and whose phenotype is unclear (26). In addition, the mutation was inherited from the mother, who has normal thyroid function, position, and size.

\section{SLC5A5/NIS Variants}

The two Tunisian patients (\#14 and \#15), born to consanguineous parents, were homozygous for the same SLC5A5 mutation, c.1183 G>A, p.G395R. In a previous study of this homozygous mutation, no perchlorate-sensitive iodide uptake was observed in COS-7 cells transfected with the mutant G395R NIS cDNA (27). Moreover, Dohan et al. have analyzed the effect of the p.G395R mutation allowing valuable insights into the structure-function and mechanistic properties of NIS
(39). Amino acid substitutions at position 395 showed that the presence of an uncharged amino acid residue with a small side chain at position 395 is required for NIS function, suggesting that glycine 395 is located in a tightly packed region of NIS. For family of patient \#14, the mother carries the variant; father's DNA was not available. The brother diagnosed also with $\mathrm{CH}$ at 8 months of age is homozygous for the mutation. For patient \#15, both parents are heterozygous as well as the healthy sibling.

\section{Unsolved Cases With "No Causative" Variants}

In four patients (\#16-19), our filter prioritization strategy identified no mutations. Among these patients, three had goiter at diagnosis and two had familial $\mathrm{CH}$, including one born to consanguineous parents.

\section{Literature Review}

We reviewed the literature by searching PubMed with the following terms: "hypothyroidism AND mutations", "hypothyroidism AND mutations AND sequencing", and "hypothyroidism AND dyshormonogenesis". We excluded articles that did not provide NM accession numbers, detailed clinical data, and/or a detailed genetic analysis. Nearly 400 patients with hypothyroidism and GIS, with or without goiter, have been evaluated using either TNGS including a mean of 14 genes or whole exome sequencing $(7,8,10-13,40,41)$. Most patients underwent thyroid sonography to assess gland position and size. Selected variants were missense mutations and nonsense mutations in coding regions and splice sites. Variants in untranslated regions or noncoding RNA and synonymous variants were disregarded. The filters used were based on minor allele frequency [mainly MAF of 0.01 or 0.02 , and 0.001 in only one study (41)] and in silico prediction tool results (usually SIFT and Polyphen-2 and less often Mutation Taster, Mutation Assessor, FATHMM, GERP score, CONDEL, and PROVEAN). Variants were classified based on frequency, in silico prediction tool results, pedigree segregation, and functional studies, as available. In one study, a score from A to $\mathrm{C}$ was used to stratify variants according to these criteria (8). The frequency of patients with mutations in known genes varied from 20 to $60 \%$ in cohorts including patients of various ethnicities (Korean, Chinese, Finnish, Italian, Saudi Arabian, Russian, and multiethnic). Table 5 recapitulates the main clinical and molecular NGS studies published so far.

\section{DISCUSSION}

We used TNGS to perform comprehensive genetic screening of a well-characterized cohort of patients with $\mathrm{CH}$ due to $\mathrm{DH}$, including patients with GIS and/or goiter at diagnosis and/or a positive perchlorate discharge test. The proportion of patients with identified disease-causing mutations was 53\%. TG mutations predominated, as described previously $(7,13)$. We found $25 \%$ of DUOX2 and DUOXA2 variants as in others European countries (42). DUOX2 mutations were uncommon in our cohort, in contrast to studies in Asians showing a prevalence of $60 \%$ (12). This could be explained by the absence 
TABLE 5 | Literature review.

\begin{tabular}{|c|c|c|c|c|c|c|c|c|}
\hline & $\begin{array}{l}\text { Park, Ann } \\
\text { Lab Med } \\
2016\end{array}$ & Lof, Thyroid, 2016 & $\begin{array}{l}\text { Jiang, Eur J Med } \\
\text { Genet, } 2016\end{array}$ & $\begin{array}{l}\text { Nicholas, J Clin } \\
\text { Endocrinol } \\
\text { Metab, } 2016\end{array}$ & $\begin{array}{l}\text { de Filippis, Hum Mol } \\
\text { Genet, } 2017\end{array}$ & Zou, JCEM, 2018 & Sun, EJE, 2018 & $\begin{array}{c}\text { Makretskaya, } \\
\text { PlosOne, } 2018\end{array}$ \\
\hline \multirow{2}{*}{$\begin{array}{l}\text { Sequencing } \\
\text { approach } \\
\text { genes }\end{array}$} & multiplexPCR & TNGS & TNGS & TNGS, WES & TNGS & WES & TNGS & $\begin{array}{l}\text { TNGS and } \\
\text { MLPA }\end{array}$ \\
\hline & $\begin{array}{l}\text { TPO, TSHR, } \\
\text { DUOX2, } \\
\text { DUOXA2, } \\
\text { NIS, PAX8 }\end{array}$ & $\begin{array}{c}\text { TG, TPO, TSHR, } \\
\text { DUOX2, IYD, NIS, } \\
\text { PDS, DUOX1, } \\
\text { NKX2-5, PAX8, TRH, } \\
\text { TRHR, TSHB }\end{array}$ & $\begin{array}{l}\text { TG, TPO, TSHR, } \\
\text { DUOX2, IYD, NIS, } \\
\text { NKX2-5, PAX8, } \\
\text { NKX2-1, FOXE1, } \\
\text { SLC26A4, GNAS }\end{array}$ & $\begin{array}{l}\text { TG, TPO, TSHR, } \\
\text { DUOX2, DUOXA2, } \\
\text { IYD, NIS, PDS }\end{array}$ & $\begin{array}{c}\text { TG, TPO, TSHR, } \\
\text { DUOX2, DUOXA2, } \\
\text { PDS, PAX8, NKX2-1, } \\
\text { FOXE1, GLIS3, JAG1 }\end{array}$ & $\begin{array}{c}\text { TG, TPO, TSHR, DUOX2, PDS, PAX8, } \\
\text { NKX2-1, SLC26A7, TSHB, CDCA8, } \\
\text { HOXB3 (mutated genes) }\end{array}$ & $\begin{array}{l}\text { TG, TPO, TSHR, } \\
\text { DUOX2, DUOXA2, } \\
\text { IYD, NIS, PDS, } \\
\text { PAX8, NKX2-1, } \\
\text { FOXE1, DIO1, }\end{array}$ & $\begin{array}{c}\text { TG, TPO, TSHR, } \\
\text { DUOX2, } \\
\text { DUOXA2, IYD, } \\
\text { NIS, PDS, PAX8, } \\
\text { NKX2-1, FOXE1, } \\
\text { NKX2-5 }\end{array}$ \\
\hline $\begin{array}{l}\text { causative genes for DH } \\
\text { or DT }\end{array}$ & 6 & 13 & 12 & 8 & 11 & WES & $\begin{array}{c}\text { DIO2, DUOX1, } \\
\text { DUOXA1, THRB, } \\
\text { THRA, GNAS, } \\
\text { SLC16A2, HHEX, } \\
\text { NKX2-5 }\end{array}$ & 12 \\
\hline \multicolumn{9}{|l|}{ Patients } \\
\hline patients with GIS & $\begin{array}{c}112(\mathrm{CH} \\
\text { newborn } \\
\text { screening) + } \\
58 \mathrm{CH} \\
\text { (outpatients, } \\
\text { adult follow- } \\
\text { up) }=170\end{array}$ & $\begin{array}{l}26 \text { (15 sporadic } \\
\text { goiter, athyreosis, } \\
\text { hypoplasia; } 11 \\
\text { families GIS, } \\
\text { athyreosis, } \\
\text { hypoplasia) }\end{array}$ & 12 & 49 & 94 (goiter, GIS) & $30 \mathrm{DH}$ (goiter, GIS) & $\begin{array}{c}110 \mathrm{CH} \text { (37 trios), } \\
21 \text { goiter, } 51 \mathrm{GIS}, \\
10 \mathrm{TD}, 28 \mathrm{NA}\end{array}$ & $243 \mathrm{CH}$ \\
\hline patients with TD & & & & & 83 & 25 TD (including $1 \mathrm{CeH}$ ) & & \\
\hline $\begin{array}{l}\text { clinical description DH/ } \\
\text { TD }\end{array}$ & $\begin{array}{l}\mathrm{CH} \text {, thyroid } \\
\text { scan and } \\
\text { ultrasound } \\
\text { for } \\
\text { outpatients } \\
\text { without data }\end{array}$ & $\begin{array}{l}\text { thyroid US if data } \\
\text { available, TG levels }\end{array}$ & $\begin{array}{c}\mathrm{CH} \text {, newborn } \\
\text { screening, ultrasonic } \\
\text { scanning }\end{array}$ & $\begin{array}{l}\mathrm{CH} \text {, newborn } \\
\text { screening, thyroid } \\
\text { imaging } \\
\text { (GIS or goiter) }\end{array}$ & $\begin{array}{c}\mathrm{CH} \text {, newborn } \\
\text { screening, thyroid US } \\
\text { and/or } \\
\text { scintigraphy }\end{array}$ & $\begin{array}{c}\mathrm{CH} \text {, newborn screening, thyroid US and/ } \\
\text { or } \\
\text { scintigraphy }\end{array}$ & $\begin{array}{l}\mathrm{CH} \text {, newborn } \\
\text { screening }\end{array}$ & $\begin{array}{c}\text { severe } \mathrm{CH}(\mathrm{TSH} \\
\text { at diagnosis } \\
>90 \mathrm{mU} / \mathrm{L})\end{array}$ \\
\hline ethnicity & Korean & Finnish & Chinese & Multiethnic origin & Italian & Saudi Arabia & Chinese & Russian \\
\hline \multicolumn{9}{|c|}{ Filters used variants analysis } \\
\hline frequency & 0.01 & 0.01 & 0.02 & 0.02 & 0.01 & & 0.01 & 0.01 \\
\hline types of variants & $\begin{array}{c}\text { missense, } \\
\text { nonsense } \\
\text { mutations in } \\
\text { coding } \\
\text { regions, } \\
\text { without } \\
\text { variants } \\
\text { UTR, non } \\
\text { coding RNA, } \\
\text { without } \\
\text { synonymous } \\
\text { variants; }\end{array}$ & $\begin{array}{l}\text { coding regions or } \\
\text { splicing exons, } \\
\text { unknown and variant } \\
<1 \% \text {; variant } \\
\text { classification with } \\
\text { selection of variants } \\
\text { with high and } \\
\text { moderate effect on } \\
\text { gene function }\end{array}$ & $\begin{array}{l}\text { no intronic and } \\
\text { synonymous, } \\
\text { "tolerated" or } \\
\text { "benign" variants } \\
\text { were excluded; rare, } \\
\text { deleterious, putative } \\
\text { deleterious variants }\end{array}$ & $\begin{array}{l}\text { variants affecting } \\
\text { protein coding } \\
\text { sequence/splicing, } \\
\text { possibly damaging } \\
\text { and } \\
\text { above }\end{array}$ & $\begin{array}{l}\text { nonsense, frameshift, } \\
\text { splice site, missense; } \\
\text { deleterious in } 5 \text { out of } \\
7 \text { algorithms of the } \\
\text { dbSNP database }\end{array}$ & $\begin{array}{l}\text { VUS: mutations if }<0.01 \text {, damaging or } \\
\text { disease } \\
\text { causing in } 3 / 4 \text { prediction tools, strict } \\
\text { segregation, biallelic: disease causing }\end{array}$ & $\begin{array}{l}\text { functional variants } \\
\text { (altering protein), } \\
\text { without UTR or } \\
\text { intergenic variants, } \\
\text { synonymous }\end{array}$ & $\begin{array}{l}\text { Benign and } \\
\text { "likely benign" } \\
\text { variants were } \\
\text { excluded }\end{array}$ \\
\hline
\end{tabular}




\begin{tabular}{|c|c|c|c|c|c|c|c|c|}
\hline & $\begin{array}{l}\text { Park, Ann } \\
\text { Lab Med } \\
2016\end{array}$ & Lof, Thyroid, 2016 & $\begin{array}{l}\text { Jiang, Eur J Med } \\
\text { Genet, } 2016\end{array}$ & $\begin{array}{l}\text { Nicholas, J Clin } \\
\text { Endocrinol } \\
\text { Metab, } 2016\end{array}$ & $\begin{array}{l}\text { de Filippis, Hum Mol } \\
\text { Genet, } 2017\end{array}$ & Zou, JCEM, 2018 & Sun, EJE, 2018 & $\begin{array}{l}\text { Makretskaya, } \\
\text { PlosOne, } 2018\end{array}$ \\
\hline & $\begin{array}{l}\text { deleterious/ } \\
\text { damaging }\end{array}$ & & & & & & & \\
\hline in silico prediction & $\begin{array}{c}\text { SIFT, } \\
\text { PolyPhen-2, } \\
\text { Mutation } \\
\text { Taster, } \\
\text { Mutation } \\
\text { Assesor, } \\
\text { FATHMM, } \\
\text { GERP } \\
\text { score }>2 \text {, } \\
\text { HGMD }\end{array}$ & Condel & $\begin{array}{l}\text { SIFT, PolyPhen-2, } \\
\text { CONDEL }\end{array}$ & $\begin{array}{l}\text { Ensembl VEP, } \\
\text { SIFT, PolyPhen-2, } \\
\text { GERP }\end{array}$ & $\begin{array}{l}\text { SIFT, Polyphen2, } \\
\text { Mutation Taster, } \\
\text { Mutation } \\
\text { Assessor, LRT, } \\
\text { FATHMM, } \\
\text { NetGen2v2.4 ESE } \\
\text { Finder } 2.0 \text { for intron } \\
\text { variants }\end{array}$ & $\begin{array}{c}\text { Mutation Taster, PolyPhen-2, SIFT, } \\
\text { PROVEAN }\end{array}$ & $\begin{array}{l}\text { Function using } \\
\text { ANNOVAR (UCSC) }\end{array}$ & $\begin{array}{l}\text { ACMG } \\
\text { guidelines }\end{array}$ \\
\hline $\begin{array}{l}\text { pedigree segregation } \\
\text { studies }\end{array}$ & $\begin{array}{l}\text { for unknown } \\
\text { mutations }\end{array}$ & in familial cases & no & when possible & in families & yes & when possible & no \\
\hline functional testing & no & $\begin{array}{l}\text { protein modeling and } \\
\text { in vitro experiments }\end{array}$ & no & $\begin{array}{l}\text { when possible, } \\
\text { protein modeling }\end{array}$ & if published & no & no & no \\
\hline \multicolumn{9}{|l|}{ Conclusions } \\
\hline $\begin{array}{l}\% \text { diagnosed patients } \\
\text { according to authors } \\
\text { criteria }\end{array}$ & $\begin{array}{l}31 \%(53 / \\
170), \\
\text { mono and } \\
\text { biallelic } \\
\text { variants }\end{array}$ & $\begin{array}{c}54.5 \% \text { (6/11 familial } \\
\text { cases), } 20 \%(3 / 15 \\
\text { sporadic cases) with } \\
\text { accurately analysis by } \\
\text { variant }\end{array}$ & $\begin{array}{l}91.7 \%(11 / 12 \\
\text { patients), mono and } \\
\text { biallelic variants }\end{array}$ & $\begin{array}{l}\text { 59\% solved cases, } \\
\text { (29/49), } 22 \% \\
\text { ambiguous cases } \\
(11 / 49), 18 \% \\
\text { unsolved cases ( } 9 / \\
\text { 49) with accurate } \\
\text { analysis by variant }\end{array}$ & $\begin{array}{l}\text { 103/177 patients with } \\
\text { a rare variant, } 5 \text { cases } \\
\text { with monogenic } \\
\text { recessive forms, } 39 \\
\text { patients with } \\
\text { oligogenic model }\end{array}$ & $\begin{array}{c}60 \%(18 / 30) \text { in DH (biallelic mutations), } \\
37.5 \%(9 / 24) \text { for TD, if HOXB3 causative: } \\
40 \% \text { (10/25) for TD }\end{array}$ & $\begin{array}{l}51.8 \%(57 / 110 \\
\text { patients) with } \\
\text { biallelic mutations, } \\
\text { recessive manner } \\
\text { of inheritance }\end{array}$ & $\begin{array}{c}37.9 \%(92 / 243) \\
\text { with variants } \\
\text { considered as } \\
\text { pathogenic/likely } \\
\text { pathogenic } \\
\text { including VUS } \\
\text { mono-and } \\
\text { biallelic }\end{array}$ \\
\hline $\begin{array}{l}\text { final conclusions of } \\
\text { article }\end{array}$ & $\begin{array}{l}\text { DUOX2: } \\
\text { frequent } \\
\text { cause of } \mathrm{CH} \\
\text { in the } \\
\text { Korean } \\
\text { population }\end{array}$ & $\begin{array}{l}\text { TNGS: cost-effective, } \\
\text { efficient, and } \\
\text { multigenic screening, } \\
\text { classification of } \\
\text { variants (based on } \\
\text { segregation, literature } \\
\text { and in vitro } \\
\text { experiments) }\end{array}$ & $\begin{array}{l}\text { high prevalence of } \\
\text { DUOX2 mutations } \\
\text { (83.3\%) in central } \\
\text { China, all patients } \\
\text { were biallelic, tri-allelic } \\
\text { or compound } \\
\text { mutations in other } \\
\text { genes }\end{array}$ & $\begin{array}{c}\text { biallelic variants } \\
\text { TPO/TG, PDS/ } \\
\text { TPO, DUOX2/TG, } \\
\text { TG+TPO : more } \\
\text { severe CH } \\
\text { phenotype, triallelic } \\
\text { variant are frequent }\end{array}$ & $\begin{array}{c}\text { Oligogenic models of } \\
\mathrm{CH} \text { with causative } \\
\text { genes, } \mathrm{CH} \text { population } \\
\text { is significantly enriched } \\
\text { with rare/low } \\
\text { frequency alleles in the } \\
11 \mathrm{CH} \text { - related genes }\end{array}$ & $\begin{array}{l}\text { TG mutations: more frequent in DH with } \\
\text { diagnostic yield of } 66 \% \text { in mutated DH } \\
\text { patients (12/18) and } 40 \% \text { for all patients } \\
\text { (12/30), TSHR more frequent in TD ( } 60 \% \\
\text { of TD mutated patients } 6 / 10 \text { and } 25 \% \\
\text { for } 6 / 24 \text { patients), SLC26A7 : new } \\
\text { candidate gene for DH? }\end{array}$ & $\begin{array}{l}\text { Diagnostic yield of } \\
51,82 \% \text { (for } \\
\text { biallelic mutations), } \\
\text { high frequency of } \\
\text { DUOX2 mutations } \\
\text { compared to } \\
\text { Caucasian } \\
\text { population }\end{array}$ & $\begin{array}{l}\text { Majority of } \\
\text { variants in } \mathrm{DH} \\
\text { genes } \\
\text { responsible for } \\
\text { severe } \mathrm{CH}\end{array}$ \\
\hline
\end{tabular}

CeH, Central hypothyroidism.

VUS, variant of incertain signifiance.

ACMG, American College of Medical Genetics and Genomics. 
of patients of Asian ethnicity in our cohort and the detailed phenotypic and molecular description and assessment of variants. The pathogenicity of each variant was carefully evaluated based on clinical data including correlations with clinical phenotypes, previously published information, availability of functional studies, in silico prediction tool results, and location of variants in regions of interest of the protein. Interestingly we detected no causative DEHAL1/IYD or SLC26A4/PDS variants, suggesting that these may be rarely responsible for $\mathrm{CH}$ due to $\mathrm{DH}$, depending usually on iodine uptake or associated with syndromic features as in the case of Pendred syndrome $(4,6)$. The proportion of patients with identified mutations differed between familial and sporadic cases (62 versus 54\%, respectively).

The biallelic TG mutations identified in patient \#2 have been reported previously and were consistent with the clinical presentation of goiter, low thyroglobulin levels, elevated iodide uptake, and normal perchlorate discharge test. Similarly, in patients \#1 and \#3, the goiter in utero or GIS with normal radionuclide uptake and nearly normal perchlorate discharge test are consistent with a $T G$ mutation. Interestingly, the perchlorate discharge test result in patient \#5 was elevated. Variable perchlorate discharge test values and partial iodide organification defects have been reported in patients with TG mutations $(43,44)$. When available, thyroglobulin values were in agreement with the molecular diagnosis, as observed in patients $\# 2$ and \#7. This finding confirms that very low thyroglobulin levels are a good indication for TG mutation screening (45).

Biallelic DUOX2 mutations explaining the $\mathrm{DH}$ were detected in a single patient (\#4), who had a goiter and a positive perchlorate discharge test. Monoallelic DUOX2 variants were identified combined with biallelic mutations in $T G$ gene. The pathogenic role of these variants and their contribution, if any, to disease severity is difficult to determine. We identified two heterozygous TPO variants (patients \#9 and \#10) as the only disease-causing candidates. DH due to monoallelic TPO variants has been reported (35). Monoallelic expression in thyroid tissue and/or other, unidentified genetic factors may explain the phenotype. Indeed, the TPO variant of the patient \#9 (c.866T $>C$, considered as deleterious through three in silico predictive algorithms) could be disease-causing if associated with another TPO variant or if there is monoallelic expression. However, given the uncertainty regarding the molecular diagnosis and the absence of functional data, the contribution to the $\mathrm{CH}$ phenotype of patients remains unclear.

We detected compound heterozygosity for two different novel DUOXA2 variants in a patient with $\mathrm{DH}$ and a partial iodide organification defect (patient \#8). DUOXA2 mutations are a rare cause of $\mathrm{DH}$, and only seven variants have been reported so far (5). Interestingly, we identified three different $P A X 8$ variants in three patients (\#11,\#12, and \#13), including two variants located in the DNA-binding domain. A single variant (p.I34N) was considered causative, based on location and familial segregation. However, p.R133W is also located in the binding domain, although a previously reported functional study found no evidence of a causative effect (25). The variant p.I34N in
PAX8 of patient \#11 was causative probably due to its involvement in transactivation of DUOX2 and TPO leading to defect of iodide organification. PAX8 mutations cause thyroid dysgenesis and some mutations were compatible with dyshormonogenesis as already described $(37,38)$.

Our literature review of studies of the molecular diagnosis of $\mathrm{CH}$ using NGS techniques including whole exome sequencing showed that differences in mutation frequencies across cohorts were chiefly ascribable to differences in ethnicity. Korean, Chinese, Finnish, Italian, Russian, or Saudi Arabian patients were studied. A single study included patients of different ethnicities (7). In two studies that distinguished between familial and sporadic cases, mutations were identified in about $54 \%$ of familial cases and $20-22 \%$ of sporadic cases $(7,8)$. No such difference was observed in our cohort. Furthermore, frequency differences across cohorts depend on the type of variant classification. Some studies determined the number of variants without differentiating variants with recessive versus dominant inheritance. Inheritance is usually recessive for variants responsible for $\mathrm{CH}$ with $\mathrm{DH}(11,12,40)$. The frequency of pathogenic variants was therefore overestimated. When identifying genetic causes of $\mathrm{CH}$, the challenge consists in using appropriate criteria to select variants for TNGS screening. Interestingly, the diagnostic yield of TNGS in our study was 53\%, in keeping with the results of previous studies that used a similar filter prioritization strategy (Table 5). Reports of digenic variants in several genes (TPO/TG, PDS/TPO, DUOX2/TG) suggest a pathogenic effect of variant accumulation, with the occurrence of $\mathrm{DH}$. Oligogenic models involving $\mathrm{CH}$-causing genes have been developed (10). We also found two or three mutated genes in several patients, although presence of a single recessive mutation was sufficient to cause $\mathrm{DH}$, without the presence of the other monoallelic variants.

Combining scintigraphy and thyroid ultrasound in the individual patient improves diagnostic accuracy and guides molecular studies. In our cohort, positive TNGS findings correlated well with thyroid radionuclide uptake and perchlorate discharge test results. TNGS after newborn screening and $\mathrm{CH}$ confirmation may be a valid strategy for rapidly obtaining the accurate diagnosis of $\mathrm{CH}$ due to $\mathrm{DH}$. TNGS, if available, may serve as a diagnostic alternative to thyroid scintigraphy, which is a time-consuming and invasive method. However, this approach should not delay treatment initiation and appropriate clinical care of patients.

In conclusion, in a well-characterized cohort of patients with $\mathrm{DH}$, our TNGS approach provided the molecular diagnosis and shed light on genetic cause in $53 \%$ of cases. Several novel mutations were detected, half of which were causative for $\mathrm{DH}$. Our analysis of the identified variants was based on both a detailed phenotypic description and an in-depth assessment of causality. TNGS is a rapid and cost-effective method for screening patients with $\mathrm{CH}$. Patients whose TNGS results fail to provide the molecular diagnosis can then be assessed using other NGS approaches, i.e., whole exome sequencing or whole genome sequencing, with the goal of identifying new candidate genes. 


\section{DATA AVAILABILITY STATEMENT}

The original contributions presented in the study are publicly available. This data can be found here: Clinical data: ClinVar accession numbers: VCV000888352, VCV000712030, VCV000631732, VCV000372358, VCV000361974, VCV000279800, VCV000265105, VCV000012706, VCV000012695, VCV000012691, VCV000007670.

\section{ETHICS STATEMENT}

The studies involving human participants were reviewed and approved by French Biomedecine Agency. Written informed consent to participate in this study was provided by the participants' legal guardian/next of kin.

\section{AUTHOR CONTRIBUTIONS}

AC and MP coordinated and instigated the study. AS, CT, GP, $\mathrm{MM}, \mathrm{SB}, \mathrm{MD}, \mathrm{RR}, \mathrm{PB}, \mathrm{MH}$, and NB provided clinical samples and data. AS and GAHC performed molecular studies. AC, AS, and GAHC analyzed the data. CB-F and SH coordinated NGS procedure. $\mathrm{PN}$ and $\mathrm{CF}$ gave bioinformatics support. $\mathrm{AC}, \mathrm{AS}$, and

\section{REFERENCES}

1. Barry Y, Bonaldi C, Goulet V, Coutant R, Léger J, Paty AC, et al. Increased incidence of congenital hypothyroidism in France from 1982 to 2012: a nationwide multicenter analysis. Ann Epidemiol (2016) 26:100-5. doi: 10.1016/j.annepidem.2015.11.005

2. Deladoëy J, Ruel J, Giguère Y, Van Vliet G. Is the incidence of congenital hypothyroidism really increasing? A 20 -year retrospective population-based study in Québec. J Clin Endocrinol Metab (2011) 96:2422-9. doi: 10.1210/ jc.2011-1073

3. Stoupa A, Kariyawasam D, Carré A, Polak M. Update of Thyroid Developmental Genes. Endocrinol Metab Clin North Am (2016) 45:243-54. doi: 10.1016/j.ecl.2016.01.007

4. Targovnik HM, Citterio CE, Rivolta CM. Iodide handling disorders (NIS, TPO, TG, IYD). Best Pract Res Clin Endocrinol Metab (2017) 31:195-212. doi: 10.1016/j.beem.2017.03.006

5. Muzza M, Fugazzola L. Disorders of $\mathrm{H} 2 \mathrm{O} 2$ generation. Best Pract Res Clin Endocrinol Metab (2017) 31:225-40. doi: 10.1016/j.beem.2017.04.006

6. Wémeau JL, Kopp P. Pendred syndrome. Best Pract Res Clin Endocrinol Metab (2017) 31:213-24. doi: 10.1016/j.beem.2017.04.011

7. Nicholas AK, Serra EG, Cangul H, Alyaarubi S, Ullah I, Schoenmakers E, et al. Comprehensive Screening of Eight Known Causative Genes in Congenital Hypothyroidism With Gland-in-Situ. J Clin Endocrinol Metab (2016) 101:4521-31. doi: 10.1210/jc.2016-1879

8. Löf C, Patyra K, Kuulasmaa T, Vangipurapu J, Undeutsch H, Jaeschke H, et al. Detection of Novel Gene Variants Associated with Congenital Hypothyroidism in a Finnish Patient Cohort. Thyroid (2016) 26:1215-24. doi: 10.1089/thy.2016.0016

9. Fan X, Fu C, Shen Y, Li C, Luo S, Li Q, et al. Next-generation sequencing analysis of twelve known causative genes in congenital hypothyroidism. Clin Chim Acta (2016) 468:76-80. doi: 10.1016/j.cca.2017.02.009

10. de Filippis T, Gelmini G, Paraboschi E, Vigone MC, Di Frenna M, Marelli F, et al. A frequent oligogenic involvement in congenital hypothyroidism. Hum Mol Genet (2017) 26:2507-14. doi: 10.1093/hmg/ddx145
MP draft and finalized the manuscript with the help of GS and DK. All authors contributed to the article and approved the submitted version.

\section{FUNDING}

AS was supported in part by an Onassis Foundation Grant and a European Society for Pediatric Endocrinology (ESPE) Research Fellowship grant. AC and MP received financial support from three corporations (EDF, Sandoz SAS, and Merck Serono France) and from the non-profit Princess Grace Foundation of Monaco. GS was supported by ESPE. Funders had no role in study design, data collection and analysis, decision to publish, or preparation of the manuscript.

\section{ACKNOWLEDGMENTS}

We thank the patients and families for their kind participation, and the IMAGINE Institute Biobank. We would like to thank Drs Brigitte Mignot, Robert Rapaport, Delphine Zenaty, Jean de Monleon, Christine Raybaud, Sylvie Soskin for clinical care of patients, for whom no causative variant was so far found.

11. Park KJ, Park HK, Kim YJ, Lee KR, Park JH, Park JH, et al. DUOX2 Mutations Are Frequently Associated With Congenital Hypothyroidism in the Korean Population. Ann Lab Med (2016) 36:145-53. doi: 10.3343/alm.2016.36.2.145

12. Sun F, Zhang JX, Yang CY, Gao GQ, Zhu WB, Han B, et al. The genetic characteristics of congenital hypothyroidism in China by comprehensive screening of 21 candidate genes. Eur J Endocrinol (2018) 178:623-33. doi: 10.1530/EJE-17-1017

13. Zou M, Alzahrani AS, Al-Odaib A, Alqahtani MA, Babiker O, Al-Rijjal RA, et al. Molecular Analysis of Congenital Hypothyroidism in Saudi Arabia: SLC26A7 Mutation Is a Novel Defect in Thyroid Dyshormonogenesis. J Clin Endocrinol Metab (2018) 103:1889-98. doi: 10.1210/jc.2017-02202

14. Stoupa A, Adam F, Kariyawasam D, Strassel C, Gawade S, Szinnai G, et al. TUBB1 mutations cause thyroid dysgenesis associated with abnormal platelet physiology. EMBO Mol Med (2018) 10:e9569. doi: 10.15252/emmm. 201809569

15. Leger J, Olivieri A, Donaldson M, Torresani T, Krude H, van Vliet G, et al. European Society for Paediatric Endocrinology consensus guidelines on screening, diagnosis, and management of congenital hypothyroidism. J Clin Endocrinol Metab (2014) 99:363-84. doi: 10.1210/jc.2013-1891

16. Alzahrani AS, Baitei EY, Zou M, Shi Y. Clinical case seminar: metastatic follicular thyroid carcinoma arising from congenital goiter as a result of a novel splice donor site mutation in the thyroglobulin gene. J Clin Endocrinol Metab (2006) 91:740-6. doi: 10.1210/jc.2005-2302

17. van de Graaf SA, Ris-Stalpers C, Veenboer GJ, Cammenga M, Santos C, Targovnik HM, et al. A premature stopcodon in thyroglobulin messenger RNA results in familial goiter and moderate hypothyroidism. J Clin Endocrinol Metab (1999) 84:2537-42. doi: 10.1210/jcem.84.7.5862

18. Caputo M, Rivolta CM, Esperante SA, Gruñeiro-Papendieck L, Chiesa A, Pellizas CG, et al. Congenital hypothyroidism with goitre caused by new mutations in the thyroglobulin gene. Clin Endocrinol (Oxf) (2007) 67:351-7. doi: 10.1111/j.1365-2265.2007.02889.x

19. Pardo V, Vono-Toniolo J, Rubio IG, Knobel M, Possato RF, Targovnik HM, et al. The p.A2215D thyroglobulin gene mutation leads to deficient synthesis and secretion of the mutated protein and congenital hypothyroidism with 
wide phenotype variation. J Clin Endocrinol Metab (2009) 94:2938-44. doi: 10.1210/jc.2009-0150

20. Pfarr N, Korsch E, Kaspers S, Herbst A, Stach A, Zimmer C, et al. Congenital hypothyroidism caused by new mutations in the thyroid oxidase 2 (THOX2) gene. Clin Endocrinol (Oxf) (2006) 65:810-5. doi: 10.1111/j.13652265.2006.02672.x

21. Targovnik HM, Esperante SA, Rivolta CM. Genetics and phenomics of hypothyroidism and goiter due to thyroglobulin mutations. Mol Cell Endocrinol (2010) 322:44-55. doi: 10.1016/j.mce.2010.01.009

22. Tonacchera M, De Marco G, Agretti P, Montanelli L, Di Cosmo C, Freitas Ferreira AC, et al. Identification and functional studies of two new dualoxidase 2 (DUOX2) mutations in a child with congenital hypothyroidism and a eutopic normal-size thyroid gland. J Clin Endocrinol Metab (2009) 94:430914. doi: $10.1210 / j c .2009-0426$

23. Brust ES, Beltrao CB, Watanabe T. New heterozygous mutations in thyroglobulin gene in patients with congenital hypothyroidism. Endoc Rev (2011). The endocrine society's 93rd annual meeting.

24. Lanzerath K, Bettendorf M, Haag C, Kneppo C, Schulze E, Grulich-Henn J. Screening for Pax8 mutations in patients with congenital hypothyroidism in South-West Germany. Horm Res (2006) 66:96-100. doi: 10.1159/000093799

25. Vincenzi M, Camilot M, Ferrarini E, Teofoli F, Venturi G, Gaudino R, et al. Identification of a novel pax8 gene sequence variant in four members of the same family: from congenital hypothyroidism with thyroid hypoplasia to mild subclinical hypothyroidism. BMC Endocr Disord (2014) 14. doi: 10.1186/ 1472-6823-14-69

26. Fu C, Chen R, Zhang S, Luo S, Wang J, Chen Y, et al. PAX8 pathogenic variants in Chinese patients with congenital hypothyroidism. Clin Chim Acta (2015) 450:322-6. doi: 10.1016/j.cca.2015.09.008

27. Kosugi S, Bhayana S, Dean HJ. A novel mutation in the sodium/iodide symporter gene in the largest family with iodide transport defect. J Clin Endocrinol Metab (1999) 84:3248-53. doi: 10.1210/jcem.84.9.5971

28. Di Jeso B, Arvan P. Thyroglobulin From Molecular and Cellular Biology to Clinical Endocrinology. Endocr Rev (2016) 37:2-36. doi: 10.1210/er.2015-1090

29. Kosugi S, Okamoto H, Tamada A, Sanchez-franco F. A Novel Peculiar Mutation in the Sodium/Iodide Symporter Gene in Spanish Siblings with Iodide Transport Defect. J Clin Endocrinol Metab (2002) 87:3830-6. doi: 10.1210/jcem.87.8.8767

30. Moreno J, Bikker H, Kempers M, van Trotsenburg P, Baas F, de Vijlder J, et al. Inactivating mutations in the gene for thyroid oxidase 2 (thox2) and congenital hypothyroidism. N Engl J Med (2002) 347:95-102. doi: 10.1056/ NEJMoa012752

31. Grasberger H, Refetoff S. Identification of the maturation factor for dual oxidase. J Biol Chem (2006) 281:18269-72. doi: 10.1074/jbc.C600095200

32. Deladoëy J, Pfarr N, Vuissoz JM, Parma J, Vassart G, Biesterfeld S, et al. Pseudodominant Inheritance of Goitrous Congenital Hypothyroidism Caused by TPO Mutations: Molecular and in Silico Studies. J Clin Endocrinol Metab (2008) 93:627-33. doi: 10.1210/jc.2007-2276

33. Poleev A, Okladnova O, Musti AM, Schneider S, Royer-Pokora B, Plachov D. Determination of functional domains of the human transcription factor PAX8 responsible for its nuclear localization and transactivating potential. Eur $J$ Biochem (1997) 247:860-9. doi: 10.1111/j.1432-1033.1997.00860.x

34. Targovnik HM, Medeiros-Neto G, Varela V, Cochaux P, Wajchenberg BL, Vassart G. A nonsense mutation causes human hereditary congenital goiter with preferential production of a 171-nucleotide-deleted thyroglobulin ribonucleic acid messenger. J Clin Endocrinol Metab (1993) 77:210-5. doi: 10.1210/jcem.77.1.8325944
35. Fugazzola L, Cerutti N, Mannavola D, Vannucchi G, Fallini C, Persani L, et al. Monoallelic expression of mutant thyroid peroxidase allele causing total iodide organification defect. J Clin Endocrinol Metab (2003) 88:3264-71. doi: 10.1210/jc.2002-021377

36. Ramos HE, Carré A, Chevrier L, Szinnai G, Tron E, Cerqueira TL, et al. Extreme phenotypic variability of thyroid dysgenesis in six new cases of congenital hypothyroidism due to PAX8 gene loss-of-function mutations. Eur J Endocrinol (2014) 171:499-507. doi: 10.1530/EJE-13-1006

37. Jo W, Ishizu K, Fujieda K, Tajima T. Congenital Hypothyroidism Caused by a PAX8 Gene Mutation Manifested as Sodium/Iodide Symporter Gene Defect. J Thyroid Res (2010) 2010:619013. doi: 10.4061/2010/619013

38. Meeus L, Gilbert B, Rydlewski C, Parma J, Roussie AL, Abramowicz M, et al. Characterization of a novel loss of function mutation of PAX8 in a familial case of congenital hypothyroidism with in-place, normal-sized thyroid. J Clin Endocrinol Metab (2004) 89:4285-91. doi: 10.1210/jc.2004-0166

39. Dohán O, Gavrielides MV, Ginter C, Amzel LM, Carrasco N. Na(+)/I (-) symporter activity requires a small and uncharged amino acid residue at position 395. Mol Endocrinol (2002) 16:1893-902. doi: 10.1210/me.20020071

40. Jiang $\mathrm{H}, \mathrm{Wu}$ J, Ke S, Hu Y, Fei A, Zhen $\mathrm{Y}$, et al. High prevalence of DUOX2 gene mutations among children with congenital hypothyroidism in central China. Eur J Med Genet (2016) 59:526-31. doi: 10.1016/ j.ejmg.2016.07.004

41. Makretskaya N, Bezlepkina O, Kolodkina A, Kiyaev A, Vasilyev EV, Petrov V, et al. High frequency of mutations in "dyshormonogenesis genes" in severe congenital hypothyroidism. PloS One (2018) 13:e0204323. doi: 10.1371/ journal.pone.0204323

42. Peters C, Nicholas AK, Schoenmakers E, Lyons G, Langham S, Serra EG, et al. DUOX2/DUOXA2 Mutations Frequently Cause Congenital Hypothyroidism that Evades Detection on Newborn Screening in the United Kingdom. Thyroid (2019) 29:790-801. doi: 10.1089/thy.2018.0587

43. Niepomniszcze H, Medeiros-Neto GA, Refetoff S, Degroot LJ, Fang VS. Familial goitre with partial iodine organification defect, lack of thyroglobulin, and high levels of thyroid peroxidase. Clin Endocrinol (Oxf) (1977) 6:27-39. doi: 10.1111/j.1365-2265.1977.tb01993.x

44. Niu DM, Hsu JH, Chong KW, Huang CH, Lu YH, Kao CH, et al. Six new mutations of the thyroglobulin gene discovered in taiwanese children presenting with thyroid dyshormonogenesis. J Clin Endocrinol Metab (2009) 94:5045-52. doi: 10.1210/jc.2009-0646

45. Targovnik HM, Citterio CE, Rivolta CM. Thyroglobulin gene mutations in congenital hypothyroidism. Horm Res Paediatr (2011) 75:311-21. doi: $10.1159 / 000324882$

Conflict of Interest: The authors declare that the research was conducted in the absence of any commercial or financial relationships that could be construed as a potential conflict of interest.

Copyright (ㄷ) 2021 Stoupa, Al Hage Chehade, Chaabane, Kariyawasam, Szinnai, Hanein, Bole-Feysot, Fourrage, Nitschke, Thalassinos, Pinto, Mnif, Baron, De Kerdanet, Reynaud, Barat, Hachicha, Belguith, Polak and Carré. This is an open-access article distributed under the terms of the Creative Commons Attribution License (CC BY). The use, distribution or reproduction in other forums is permitted, provided the original author(s) and the copyright owner(s) are credited and that the original publication in this journal is cited, in accordance with accepted academic practice. No use, distribution or reproduction is permitted which does not comply with these terms. 